\title{
ASSESSMENT OF PATELLAR HEIGHT MEASUREMENT METHODS FROM DIGITAL RADIOGRAPHY
}

Guilherme Conforto Gracitelli', Rafael Pierami', Thomaz Antônio Tonelli', Guilherme Guadagnini Falótico², Flavio Duarte Silva ${ }^{3}$, Gilberto Yoshinobu Nakama ${ }^{4}$, Carlos Eduardo da Silveira Franciozi ${ }^{5}$, Antônio Altenor Bessa de Queiroz ${ }^{6}$, Mario Carneiro Filho ${ }^{7}$

\section{ABSTRACT}

Objective: To analyze the most common methods for measuring patellar height and the impact of observer experience in correlations with the other observers using digital radiography. Methods: Sixty digital radiographs of the knee in lateral view were analyzed by four observers: a physician in the second year of medical residence in orthopedics (R2); a physician in the third year of medical residence in orthopedics (R3); an orthopedic surgeon who was a specialist in knee surgery (SK); and a radiologist who was a specialist in musculoskeletal radiology (SR). The indices used were: Insall-Salvati (IS), Blackburne-Peel (BP), Caton-Deschamps (CD) and modified Insall-Salvati
(ISM). The interobserver agreement was calculated using the kappa coefficient $(\kappa)$. Results: The highest correlation coefficients were found when using the IS method followed by the CD method. The worst correlation was observed in the ISM method. The highest interobserver agreement was found between the orthopedic surgeon specializing in knee surgery and the radiologist specializing in musculoskeletal radiology, for the four measurement methods used. Conclusion: Using digital radiography, the Insall-Salvati and Caton-Deschamps indexes presented the highest interobserver agreement, and this was also positively influenced by the observer's level of experience.

Keywords - Knee; Patella; Radiographic Image Enhancement

\section{INTRODUCTION}

Today, use of digital radiography is becoming increasingly widespread. The advantages of this radiography system over conventional systems lie in the speed and precision of the technique, the elimination of printing and its cost, along with the ease of image display. Digital radiography enables reduction of the X-ray dosage, which can be adjusted so that the image has a convenient signal-noise ratio, thereby diminishing the amount of radiation absorbed by the patient. Image processing is facilitated, using digital techniques of increased contrast and histogram equalization, as is image acquisition, storage and retrieval from electronic databases. The disadvantages of such systems are the dependence on access to electronic databases, the cost of the software, the limitations of applications based on the software design and, especially, the lack of studies validating and evaluating measurement methods that are already well-established with conventional radiography, such as the various indices for patellar height ${ }^{(1-4)}$.

Patellar height is a parameter that is increasingly used, given that it expands the knowledge of knee biomechanics and the physiopathology of knee ailments and their respective treatments. The importance of the patella for the knee, in addition to its protective and esthetic characteristics, lies mainly in the way in which it increases the lever arm of the extensor apparatus, thereby increasing quadriceps strength by up to $50 \%{ }^{(5)}$.

1 - Third-year Resident Physician in the Department of Orthopedics and Traumatology, EPM/UNIFESP, São Paulo, SP, Brazil.

2 - Orthopedist in the Department of Orthopedics and Traumatology, EPM/UNIFESP, São Paulo, SP, Brazil.

3 - Radiologist in the Department of Imaging Diagnostics, EPM/UNIFESP, São Paulo, SP, Brazil.

4 - Orthopedist in the Knee Group, Department of Orthopedics and Traumatology, EPM/UNIFESP, São Paulo, SP, Brazil.

5 - PhD; Orthopedists in the Knee Group, Department of Orthopedics and Traumatology, EPM/UNIFESP, São Paulo, SP, Brazil.

6 - MSc; Orthopedist in the Knee Group, Department of Orthopedics and Traumatology, EPM/UNIFESP, São Paulo, SP, Brazil.

7 - PhD; Affiliated Professor and Head of the Knee Group, Department of Orthopedics and Traumatology, EPM/UNIFESP, São Paulo, SP, Brazil.

Work performed in the Department of Orthopedics and Traumatology, Paulista School of Medicine (EPM), Federal University of São Paulo (UNIFESP).

Correspondence: Rua Borges Lagoa 783, 5ªndar, 04038901 São Paulo, SP. E-mail: ggracitelli@gmail.com - cacarlos66@hotmail.com

Work received for publication: July 6, 2010; accepted for publication: August 4, 2011. 
The patellar height can be correlated with various clinical situations that affect patellofemoral joint functioning. It has been recognized that a high patella is associated with greater risk of chondromalacia and patellofemoral dislocation $^{(6-9)}$, while a low patella is associated with greater risk of patellofemoral osteoarthritis, Osgood-Schlatter disease and limited range of knee motion ${ }^{(5,10)}$.

Over the course of time, a variety of methods have been proposed as ways of defining the concept of patellar height, usually involving a ratio between radiographic parameters ${ }^{(11-18)}$. A few studies have compared the different indices for patellar height and have made critical analyses regarding their reproducibility, applicability and results. The impact of skeletal maturity has sometimes also been assessed. In many cases, these analyses have used radiographs on abnormal or diseases knees ${ }^{(15,17,19-22)}$. Even fewer studies have compared inter-observer concordance between the commonest methods for measuring patellar height in normal knees, i.e. the Insall-Salvati (IS), modified Insall-Salvati (ISM), Blackburne-Peel (BP) and Caton-Deschamps (CD) methods ${ }^{(19,21)}$.

The objective of this study was to analyze the commonest methods for measuring patellar height in relation to inter-observer concordance, and the impact of the observer's experience on correlations with other evaluators, using digital radiography.

\section{MATERIALS AND METHODS}

Sixty digital radiographs of knees in lateral view, from patients without complaints relating to the knee examined ( 30 males and 30 females) were evaluated. This study was approved by the Ethics Committee of the Federal University of São Paulo, and all the patients signed a free and informed consent statement.

The radiographs were produced with the knee flexed at $30^{\circ}$, which was controlled by means of a triangular cushioned wooden pad at an angle of $30^{\circ}$. A piece of film measuring $30 \times 40 \mathrm{~cm}$ was placed at a distance of one meter from the $\mathrm{x}$-ray tube of the digital radiographic machine, and lateral-view images were then obtained.

Radiographs of skeletally immature patients and from patients with knee osteoarthritis of grade greater than or equal to 2 in anteroposterior view, according to the Ahlback classification, ${ }^{(23)}$ as modified by Keyes et $\mathrm{al}^{(24)}$, were excluded. In addition, we excluded patients with known sequelae from Osgood-Schlatter disease and Sinding-Larsen-Johansson disease, patients with patellofemoral syndromes and patients with previous knee surgery that had given rise to direct or indirect alterations to the anterior tuberosity of the tibia.

The radiographs were analyzed by four observers with different levels of experience: one physician in the first year of medical residence in orthopedics (R1); one physician in the third year of medical residence in orthopedics (R3); one orthopedist who was a specialist in knees, certified by the Brazilian Society of Knee Surgery (EJ); and one radiologist who was a specialist in the musculoskeletal field, certified by the Brazilian College of Radiology (ER).

An electronic document was set up to standardize the measurements according to the method applied, which could be used by the investigators at any time during the evaluation.

The Insall-Salvati index consists of the ratio $\mathrm{CT} / \mathrm{CP}$, where $\mathrm{CT}$ is the length of the patellar tendon measured along its posterior or deep surface, from its origin at the inferior pole of the patella to its insertion in the tibial tubercle, and $\mathrm{CP}$ is the greatest diagonal length of the patella ${ }^{(11)}$.

The Blackburne and Peel index ${ }^{(13)}$ consists of the ratio $A / B$, where $A$ is the perpendicular height of the distal part of the joint surface of the patella, up to a line projected anteriorly to the surface of the tibial plateau, and $\mathrm{B}$ is the length of the joint surface of the patella.

The Caton-Deschamps index consists of the ratio $\mathrm{AT} / \mathrm{AP}$, in which AT is the distance from the lower edge of the joint surface of the patella to the anterosuperior angle of the tibia, and AP is the length of the joint surface of the patella ${ }^{(12)}$.

The modified Insall-Salvati index consists of the ratio $\mathrm{C} / \mathrm{D}$, in which $\mathrm{C}$ is the distance from the lower edge of the joint surface of the patella to the insertion of the posterior or deep surface of the patellar tendon in the tibial tubercle, and $\mathrm{D}$ is the length of the joint surface of the patella ${ }^{(14)}$.

The four observers obtained the Insall-Salvati (IS), Blackburne-Peel (BP), Caton-Deschamps (CD) and modified Insall-Salvati (ISM) indices from digital radiographs with $100 \%$ zoom, on slides in PowerPoint $\left(\right.$ Microsoft $\left.^{\circledR}\right)$, using the Universal Desktop Ruler software (AVPSoft ${ }^{\circledR}$ ) for measurements.

Descriptive analysis was performed on the study sample. The kappa correlation coefficient was used as the statistical analysis method. The kappa concordance coefficient provides paired concordance 
proportions between the observers, which may randomly be correct. The kappa values can range from -1 to +1 ; values between -1 and 0 indicate that the concordance observed was less than what would be expected by chance; 0 indicates a concordance level that would be expected by chance; and +1 indicates total concordance. In general, kappa values lower than 0.5 are considered to be unsatisfactory; values between 0.5 and 0.75 are considered to be satisfactory and adequate; and values greater than 0.75 are considered to be excellent ${ }^{(25-27)}$.

The parameters for normality, which were used in conformity with the literature, are described in Table 1. The stratification of kappa values was done in terms of classifications as low, normal and high patella, for each observer, according to the index used.

Table 1 - Insall-Salvati (IS), Blackburne-Peel (BP), Caton-Deschamps (CD) and modified Insall-Salvati (ISM).

\begin{tabular}{c|c|c|c}
\hline Method & Low patella & Normal patella & High patella \\
\hline IS & $<0.80$ & $0.8-1.2$ & $>1.2$ \\
\hline BP & $<0.80$ & $0.8-1.0$ & $>1.0$ \\
\hline CD & 0.60 & $0.60-1.30$ & $>1.3$ \\
\hline ISM & ------ & ----- & $>2.0$ \\
\hline
\end{tabular}

\section{RESULTS}

The mean age of the patients evaluated was 37.82 years. Table 2 demonstrates the values obtained from the analysis.

Table 3 demonstrates the kappa correlation coefficients between the four observers for the Insall-Salvati (IS), Blackburne-Peel (BP), Caton-Deschamps (CD) and modified Insall-Salvati (ISM) methods.

The highest inter-observer correlation coefficients were obtained using the IS method, followed by the CD method. The worst correlation was observed using the ISM method, followed by the BP method.

The highest inter-observer concordance was obtained between the orthopedist who was a knee specialist and the radiologist who was a musculoskeletal specialist, for the four measurement methods used.

\section{DISCUSSION}

The Insall-Salvati (IS) method uses the length of the patellar ligament in relation to the length of the patella ${ }^{(11)}$. The patellar morphology and morphological differences in the anterior tuberosity of the tibia (ATT) directly affect the measurements made using this
Table 2 - Second-year resident in orthopedics (R2), third-year resident in orthopedics (R3), orthopedist who was a knee specialist (EJ), radiologist who was musculoskeletal specialist (ER). Insall-Salvati (IS), BlackburnePeel (BP), Caton-Deschamps (CD): 1 - low patella; 2 - normal patella; 3 - high patella. Modified Insall-Salvati (ISM): 4 - non-high patella; 5 - high patella.

\begin{tabular}{|c|c|c|c|c|c|c|c|c|c|c|c|c|c|c|c|}
\hline \multicolumn{4}{|c|}{ IS } & \multicolumn{4}{|c|}{ BP } & \multicolumn{4}{|c|}{ CD } & \multicolumn{4}{|c|}{ ISM } \\
\hline EJ & ER & $\overline{\mathrm{R} 2}$ & \begin{tabular}{|l|l} 
R3 \\
\end{tabular} & $\mathrm{EJ}$ & ER & R2 & R3 & EJ & ER & R2 & R3 & EJ & ER & R2 & R3 \\
\hline 2 & 2 & 2 & 2 & 2 & 1 & 2 & 3 & 2 & 2 & 2 & 2 & 5 & 4 & 5 & 4 \\
\hline 2 & 2 & 2 & 2 & 3 & 3 & 3 & 3 & 2 & 3 & 3 & 2 & & & & \\
\hline 2 & 2 & 2 & 2 & 2 & 2 & 2 & 3 & 2 & 2 & 2 & 2 & 4 & 4 & 5 & 4 \\
\hline 3 & 3 & 3 & 3 & 2 & 3 & 3 & 3 & 2 & 2 & 3 & 2 & 5 & 4 & 5 & 4 \\
\hline 2 & 2 & 2 & 2 & 1 & 1 & 2 & 3 & 2 & 2 & 2 & 2 & 4 & 4 & 4 & 4 \\
\hline 2 & 3 & 2 & 3 & 2 & 2 & 2 & 3 & 2 & 2 & 2 & 2 & 4 & 4 & 4 & 4 \\
\hline 2 & 3 & 3 & 3 & 1 & 2 & 2 & 3 & 2 & 2 & 2 & 2 & 4 & 4 & 4 & 4 \\
\hline 2 & 2 & 2 & 2 & 1 & 1 & 1 & 3 & 1 & 2 & 2 & 2 & 4 & 4 & 4 & 4 \\
\hline 2 & 2 & 2 & 3 & 3 & 3 & 2 & 3 & 2 & 2 & 2 & 2 & 4 & 4 & 4 & 4 \\
\hline 3 & 3 & 3 & 3 & 3 & 2 & 3 & 3 & 2 & 2 & 3 & 2 & 5 & 4 & 5 & 4 \\
\hline 3 & 2 & 2 & 2 & 1 & $\frac{4}{1}$ & 2 & 3 & 2 & 2 & 2 & 2 & 4 & 4 & 4 & 4 \\
\hline 2 & 2 & 2 & 2 & 2 & 1 & 2 & 3 & 2 & 2 & 2 & 2 & 4 & 4 & 4 & 4 \\
\hline & 2 & 2 & 2 & 1 & 1 & 1 & 3 & 1 & 2 & 2 & 1 & 4 & 4 & 4 & 4 \\
\hline 3 & 3 & 3 & 3 & 1 & 1 & 1 & 3 & 2 & 2 & 2 & 1 & 5 & 4 & 5 & 4 \\
\hline & 3 & 3 & 3 & 2 & 2 & 2 & 3 & 2 & 2 & 2 & 2 & 5 & 4 & 5 & 4 \\
\hline$\overline{2}$ & 2 & 2 & 2 & 2 & 2 & 2 & 3 & 2 & 2 & 2 & 2 & 4 & 4 & 5 & 4 \\
\hline & 2 & 2 & 3 & 1 & 1 & 2 & 3 & 2 & 2 & 2 & 2 & 4 & 4 & 4 & 4 \\
\hline & 2 & 2 & 2 & 2 & 3 & 1 & 3 & 2 & 3 & 2 & 2 & 5 & 4 & 4 & 4 \\
\hline 3 & 3 & 3 & 3 & 2 & 2 & 3 & 3 & 2 & 2 & 2 & 2 & 5 & 5 & 5 & 4 \\
\hline 2 & 2 & 2 & 3 & 3 & 2 & 2 & 3 & 2 & 2 & 2 & 2 & 5 & 4 & 5 & 4 \\
\hline 2 & 2 & 2 & 3 & 2 & 3 & 3 & 3 & 2 & 3 & 2 & 2 & 5 & 4 & 5 & 4 \\
\hline 3 & 3 & 3 & 3 & 2 & 1 & 2 & 3 & 2 & 2 & 2 & 2 & 4 & 4 & 5 & 4 \\
\hline 2 & 2 & 2 & 2 & 2 & 2 & 2 & 3 & 2 & 2 & 2 & 2 & 4 & 4 & 4 & 4 \\
\hline 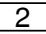 & 2 & 2 & 2 & 1 & 1 & 2 & 3 & 2 & 2 & 2 & 2 & 4 & 4 & 4 & 4 \\
\hline 3 & 3 & 3 & 3 & 2 & 2 & 3 & 3 & 2 & 2 & 2 & 2 & 5 & 4 & 4 & 4 \\
\hline & 2 & 2 & 3 & 2 & 2 & 3 & 3 & 2 & 2 & 3 & 2 & 4 & 4 & 5 & 4 \\
\hline 3 & 2 & 3 & 3 & 1 & 1 & 2 & 3 & \begin{tabular}{|l|}
2 \\
\end{tabular} & 2 & 2 & 2 & 4 & 4 & 4 & 4 \\
\hline 3 & 3 & 3 & 3 & 2 & 1 & 2 & 3 & 2 & 2 & 2 & 2 & 4 & 4 & 4 & 4 \\
\hline 2 & 2 & 2 & 3 & 3 & 2 & 2 & 3 & 2 & 2 & 2 & 2 & 5 & 4 & 4 & 4 \\
\hline$\overline{2}$ & 2 & 3 & 3 & 2 & 2 & 2 & 3 & 2 & 2 & 2 & 2 & 5 & 4 & 4 & 4 \\
\hline 3 & 3 & 3 & 3 & 2 & 2 & 1 & 3 & 2 & 2 & 2 & 2 & 4 & 4 & 4 & 4 \\
\hline 2 & 2 & 3 & 3 & 2 & 3 & 3 & 3 & 2 & 2 & 2 & 2 & 5 & 4 & 5 & 4 \\
\hline 2 & 2 & 3 & 2 & 2 & 2 & 3 & 3 & 2 & 2 & $\frac{4}{2}$ & 2 & 5 & 4 & 5 & 4 \\
\hline$\overline{3}$ & 3 & 3 & 3 & 3 & 3 & 3 & 3 & 2 & 2 & 3 & 2 & 5 & 5 & 5 & 4 \\
\hline 3 & 3 & 3 & 3 & 2 & 2 & 2 & 3 & 2 & 2 & 2 & 2 & 5 & 4 & 5 & 4 \\
\hline & 2 & 3 & 3 & 1 & 1 & 1 & 3 & 2 & 2 & 3 & 2 & 4 & 4 & 4 & 4 \\
\hline 3 & 3 & 2 & 3 & 2 & 2 & 2 & 3 & 2 & 2 & 2 & 2 & 4 & 4 & 4 & 4 \\
\hline & 3 & 2 & 3 & 2 & 2 & 3 & 3 & 2 & 2 & 2 & 2 & 4 & 4 & 4 & 4 \\
\hline 2 & 2 & 2 & 3 & 2 & 2 & 2 & 3 & 2 & 2 & 2 & 2 & 4 & 4 & 4 & 4 \\
\hline & 2 & 2 & 3 & 2 & 2 & 2 & 3 & 2 & 2 & 2 & 2 & 4 & 4 & 4 & 4 \\
\hline & 3 & 3 & 3 & 2 & 2 & 3 & 3 & 2 & 2 & 2 & 2 & 4 & 4 & 4 & 4 \\
\hline & 2 & 2 & 2 & 1 & 1 & 1 & 3 & 2 & 2 & 2 & 2 & 4 & 4 & 4 & 4 \\
\hline & 3 & 3 & 3 & 3 & 2 & 3 & 3 & 2 & 2 & 2 & 2 & 5 & 4 & 5 & 4 \\
\hline 2 & 2 & 2 & 3 & 2 & 2 & 2 & 3 & 2 & 2 & 2 & 2 & 5 & 4 & 4 & 4 \\
\hline 3 & 3 & 3 & 3 & 3 & 3 & 3 & 3 & 3 & 3 & 3 & 2 & 5 & 5 & 5 & 4 \\
\hline 2 & 2 & 2 & 3 & 2 & 2 & 2 & 3 & 2 & 2 & 2 & 2 & 5 & 4 & 5 & 4 \\
\hline 3 & 3 & 3 & 3 & 3 & 1 & 2 & 3 & 3 & 2 & 2 & 2 & 5 & 5 & 5 & 4 \\
\hline 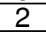 & 2 & 3 & 3 & 1 & 1 & 2 & 3 & 2 & 2 & 2 & 2 & 4 & 4 & 4 & 4 \\
\hline 2 & 2 & 2 & 3 & 2 & 3 & 1 & 3 & 2 & 3 & 2 & 2 & 4 & 4 & 4 & 4 \\
\hline 7 & 2 & \begin{tabular}{|l}
2 \\
\end{tabular} & 3 & 1 & 1 & 1 & 3 & 2 & 2 & 2 & 1 & 4 & 4 & 4 & 4 \\
\hline & 3 & 3 & 3 & 1 & 1 & 2 & 3 & 2 & 2 & 2 & 2 & 4 & 4 & 4 & 4 \\
\hline 2 & 2 & 2 & 2 & 2 & 2 & 2 & 3 & 2 & 2 & 2 & 2 & 4 & 4 & 4 & 4 \\
\hline & 2 & 2 & 3 & 3 & 3 & 3 & 3 & 2 & 2 & 2 & 2 & 4 & 4 & 4 & 4 \\
\hline 3 & 2 & 2 & 2 & 3 & 2 & 3 & 3 & 2 & 2 & 2 & 2 & 4 & 4 & 4 & 4 \\
\hline 3 & 3 & 3 & 3 & 2 & 2 & 2 & 3 & 2 & 2 & 2 & 2 & 4 & 4 & 5 & 4 \\
\hline 2 & 3 & \begin{tabular}{|l}
2 \\
\end{tabular} & 2 & 2 & 2 & 2 & 3 & 2 & 2 & 2 & 2 & 5 & 4 & 4 & 4 \\
\hline 3 & 3 & 3 & 3 & 3 & 3 & 2 & 3 & 2 & 2 & 2 & 2 & 5 & 5 & 5 & 4 \\
\hline 3 & 3 & 3 & 3 & 2 & 2 & 2 & 3 & 2 & 2 & 2 & 2 & 5 & 5 & 5 & 4 \\
\hline 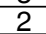 & 3 & \begin{tabular}{|l}
2 \\
\end{tabular} & 3 & $\frac{4}{1}$ & $\frac{4}{1}$ & 2 & 3 & 2 & 2 & 2 & 2 & 4 & 4 & 4 & 4 \\
\hline 2 & 3 & 3 & 3 & 2 & 1 & 2 & 3 & 2 & 2 & 2 & 2 & 4 & 4 & 4 & 4 \\
\hline
\end{tabular}

Table 3 - Inter-observer kappa coefficient.

\begin{tabular}{c|c|c|c|c}
\hline & IS & BP & CD & ISM \\
\hline EJ vs. ER & 0.808 & 0.769 & 0.824 & 0.696 \\
\hline EJ vs. R1 & 0.796 & 0.608 & 0.696 & 0.619 \\
\hline EJ vs. R3 & 0.726 & 0.426 & 0.619 & 0.268 \\
\hline ER vs. R1 & 0.703 & 0.574 & 0.749 & 0.637 \\
\hline ER vs. R3 & 0.809 & 0.366 & 0.707 & 0.519 \\
\hline R1 vs. R3 & 0.603 & 0.452 & 0.609 & 0.433
\end{tabular}

First-year resident physician in orthopedics (R1); third-year resident physician in orthopedics (R3); orthopedist who was a knee specialist, certified by the Brazilian Society of Knee Surgery (EJ); and radiologist who was specialist in the musculoskeletal field, certified by the Brazilian Collage of Radiology (ER) 
$\operatorname{method}^{(13)}$. Exclusion of patients with sequelae from Osgood-Schlatter and Sinding-Larsen-Johansson, and with previous surgery on the knee, helps to increase the concordance when using this index. Better definition of the soft tissues using digital radiography may also contribute towards improving the correlation found ${ }^{(4)}$.

Grelsamer and Meadows ${ }^{(14)}$ developed the modified Insall-Salvati (ISM) method based on the length of the joint surface. Difficulty in identifying this parameter is considered to be the main measurement bias. In the present study, the concordance obtained was similar to what is seen in the literature, and it was the lowest among all the methods evaluated. Digital radiography seems not to present greater details for this anatomical reference ${ }^{(14,19,21,22)}$.

The Blackburne-Peel (BP) method exchanges the reference point of the ATT for the joint surface of the tibial plateau, while keeping the joint surface of the patella. Although Berg et $\mathrm{al}^{(19)}$ found that this was the most accurate and reproducible method in conjunction with the IS index, and Seil et $\mathrm{al}^{(21)}$ ranked it as the second most reproducible method in conjunction with the IS index, we did not obtain similar results in our analysis, such that it was only better than the ISM index. Lack of definition of the reference line of the tibial plateau, such as which condyle to use as the reference, or whether this line runs parallel to the joint surface or perpendicular to the long axis of the tibia, contributes towards lower concordance with this method ${ }^{(4)}$.

The method of Caton et $\mathrm{al}^{(12)}$, which uses the joint

\section{REFERENCES}

1. Nobrega Al, editor. Tecnologia radiológica e diagnóstico por imagem. São Paulo: Editora Difusão; 2006.

2. Jamali AA. Digital templating and preoperative deformity analysis with standard imaging software. Clin Orthop Relat Res. 2009;467(10):695-704.

3. Bono JV. Digital templating in total hip arthroplasty. J Bone Joint Surg Am. 2004;86(Suppl 2):118-22.

4. Phillips CL, Silver DA, Schranz PJ, Mandalia V. The measurement of patellar height: a review of the methods of imaging. J Bone Joint Surg Br. 2010;92(8):1045-53.

5. Rogers BA, Thornton-Bott P, Cannon SR, Briggs TW. Interobserver variation in the measurement of patellar height after total knee arthroplasty. J Bone Joint Surg Br. 2006;88(4):484-8.

6. Ali SA, Helmer R, Terk MR. Patella alta: lack of correlation between patellotrochlear cartilage congruence and commonly used patellar height ratios. AJR Am J Roentgenol. 2009;193(5):1361-6.

7. Insall J, Falvo KA, Wise DW. Chondromalacia patellae. A prospective study. J Bone Joint Surg Am. 1976;58(1):1-8.

8. Insall J, Goldberg V, Salvati E. Recurrent dislocation and the high-riding patella. Clin Orthop Relat Res. 1972;88:67-9.

9. Moller BN, Krebs B, Jurik AG. Patellar height and patellofemoral congruence. Arch Orthop Trauma Surg. 1986;104(6):380-1.

10. Aparicio G, Abril JC, Calvo E, Alvarez L. Radiologic study of patellar height in Osgood-Schlatter disease. J Pediatr Orthop. 1997;17(1):63-6.

11. Insall J, Salvati E. Patella position in the normal knee joint. Radiology. 1971;101(1):101-4

12. Caton J, Deschamps G, Chambat P, Lerat JL, Dejour H. [Patella infera. Apropos of 128 cases]. Rev Chir Orthop Reparatrice Appar Mot. 1982;68(5):317-25.

13. Blackburne JS, Peel TE. A new method of measuring patellar height. J Bone Joint Surg Br. 1977;59(2):241-2.

14. Grelsamer RP, Meadows S. The modified Insall-Salvati ratio for assessment of patellar height. Clin Orthop Relat Res. 1992;(282):170-6. surface of the patella and the angle of the tibial plateau as references, also presents difficulty regarding identification of the joint surface, as well as presenting a certain amount of variability in defining the angle of the tibial plateau $^{(4)}$. Despite these factors, this method was the one that showed greatest concordance in the study by Seil et $\mathrm{al}^{(21)}$. In our study, it presented moderately positive concordance in all the situations evaluated.

The observer's experience was shown to be important in the present study, given that the two more experienced examiners presented higher correlation coefficients. Although this matter had previously been evaluated in concordance studies involving fracture classifications, it had not been done in studies measuring patellar height ${ }^{(28)}$.

The reliability of the IS and CD indices was congruent with what is seen in the literature, which strengthens our results. Use of computerized methods for obtaining radiographic images and measuring the patellar height seem to be an effective tool that adds precision to the measurements ${ }^{(4,21)}$.

\section{CONCLUSION}

Digital radiography for measuring the height of the patella using the methods already established with conventional radiography is a useful tool, presenting good inter-observer correlation. The Insall-Salvati and Caton-Deschamps indices presented greater concordance and this was also influenced positively by the observer's experience.

15. Hepp WR. Two new methods for determination of the height of the patella. $Z$ Orthop Ihre Grenzgeb. 1984;122(2):159-66.

16. Egund N, Lundin A, Wallengren NO. The vertical position of the patella. A new radiographic method for routine use. Acta Radiol. 1988;29(5):555-8.

17. Koshino T, Sugimoto K. New measurement of patellar height in the knees of children using the epiphyseal line midpoint. J Pediatr Orthop. 1989;9(2):216-8.

18. Portner $\mathrm{O}$, Pakzad $\mathrm{H}$. The evaluation of patellar height: a simple method. J Bone Joint Surg Am. 2011;93(1):73-80.

19. Berg EE, Mason SL, Lucas MJ. Patellar height ratios. A comparison of four measurement methods. Am J Sports Med. 1996;24(2):218-21.

20. Aparicio G, Abril JC, Albinana J, Rodriguez-Salvanes F. Patellar height ratios in children: an interobserver study of three methods. J Pediatr Orthop B. 1999;8(1):29-32.

21. Seil R, Muller B, Georg T, Kohn D, Rupp S. Reliability and interobserver variability in radiological patellar height ratios. Knee Surg Sports Traumatol Arthrosc. 2000;8(4):231-6.

22. Kadakia NR, Ilahi OA. Interobserver variability of the Insall-Salvati ratio. Orthopedics. 2003;26(3):321-3.

23. Ahlback S. Osteoarthrosis of the knee. A radiographic investigation. Acta Radiol Diagn (Stockh). 1968:(Suppl 277):7-72.

24. Keyes GW, Carr AJ, Miller RK, Goodfellow JW. The radiographic classification of medial gonarthrosis. Correlation with operation methods in 200 knees. Acta Orthop Scand. 1992;63(5):497-501.

25. Fleiss JL, Slakter MJ, Fischman SL, Park MH, Chilton NW. Inter-examiner reliability in caries trials. J Dent Res. 1979;58(2):604-9.

26. Landis JR, Koch GG. The measurement of observer agreement for categorical data. Biometrics. 1977;33(1):159-74.

27. Zou KH, Tuncali K, Silverman SG. Correlation and simple linear regression. Radiology. 2003;227(3):617-22.

28. Belloti JC, Tamaoki MJ, Franciozi CE, Santos JB, Balbachevsky D, Chap Chap E, et al. Are distal radius fracture classifications reproducible? Intra and interobserver agreement. Sao Paulo Med J. 2008;126(3):180-5. 